(C) 2005 The Japan Society of Applied Physics

\title{
High-Quality ZnO Thin Films Grown by Fast Pulsed Laser Deposition without a Buffer Layer
}

\author{
Ming-Zheng Lin, Chun-Tsung Su, Hong-Chang YAN and Ming-Yau CHERn* \\ Department of Physics, National Taiwan University, Taipei 10617, Taiwan \\ (Received December 20, 2004; accepted May 5, 2005; published July 22, 2005) \\ For the first time, a fast pulsed laser deposition (FPLD) process is presented for the epitaxial growth of $\mathrm{ZnO}$ thin films on \\ $c$-plane sapphire substrates. This process is driven by a $355 \mathrm{~nm}$ solid-state laser pulsing at $10 \mathrm{kHz}$. The $\mathrm{ZnO}$ thin films grown \\ by FPLD show a width of $\sim 10$ arcsec in the X-ray $\omega$-scan and distinct Pendellösung fringes in the $\theta-2 \theta$ scan of the $\mathrm{ZnO}(0002)$ \\ reflection. Photoluminescence spectra reveal sharp donor-bound excitons of $\sim 5 \mathrm{meV}$ width and free excitons up to $n=2$. \\ Compared to conventional (much slower) PLD, 1-20 Hz, the measurements indicate that higher quality ZnO films can be \\ grown by FPLD. In particular, despite the large lattice mismatch, such $\mathrm{ZnO}$ films were grown by FPLD directly on sapphire \\ substrates without a buffer layer. [DOI: 10.1143/JJAP.44.L995]
}

KEYWORDS: pulsed laser deposition, fast pulsed laser deposition, $\mathrm{ZnO}$, sapphire, buffer layer, Pendellösung fringes, excitons

$\mathrm{ZnO}$ semiconductors with a band gap of $3.37 \mathrm{eV}$ are widely used for applications such as piezoelectric transducers, varistors, phosphors, and transparent conducting films. The optical properties of $\mathrm{ZnO}$ are similar to those of $\mathrm{GaN}$, which has been successfully applied in short-wavelength light-emitting devices. ${ }^{1,2)}$ However, $\mathrm{ZnO}$ has a larger exciton binding energy and a higher melting temperature, $60 \mathrm{meV}$ and $1975^{\circ} \mathrm{C}$, than $\mathrm{GaN}, 28 \mathrm{meV}$ and $900^{\circ} \mathrm{C}$, respectively. ${ }^{3)}$ Therefore, it is expected that $\mathrm{ZnO}$ is a more efficient and durable material for blue and even ultraviolet light emissions. In recent years, high-quality $\mathrm{ZnO}$ films fabricated by pulsed laser deposition (PLD), ${ }^{4-6)}$ molecularbeam epitaxy (MBE), ${ }^{7-9)}$ and sputtering ${ }^{10,11)}$ showed that $\mathrm{ZnO}$ could be a promising material for the optical devices used in the short wavelength region.

The PLD method has been used to grow oxide films, including $\mathrm{ZnO}$, by many researchers. ${ }^{4-6)}$ The lasers for this method are usually operated at low pulse repetition rates, 1$20 \mathrm{~Hz}$, yet the energy per pulse is high, $\sim 100 \mathrm{~mJ} /$ pulse. Hence, the amount of species arriving at the substrates could be instantaneously large, causing the condensation of undesired particulates in the films and introducing various types of intrinsic defect. ${ }^{4,6)}$ Therefore, the film quality can be poor without carefully controlling the growth parameters. In this letter, we developed a new method of growing $\mathrm{ZnO}$ films: fast pulsed laser deposition (FPLD). A fast $10 \mathrm{kHz}$ pulsed laser was used to irradiate the $\mathrm{ZnO}$ target. At the same time, atomic oxygen was introduced into a growth chamber using an RF atom source to assist the film growth. The combination of the two techniques sufficiently prevented the generation of particulates, resulting in the improvement of the quality of the epitaxial $\mathrm{ZnO}$ films as indicated by several structural and physical measurements.

$\mathrm{ZnO}$ films were grown on $\mathrm{Al}_{2} \mathrm{O}_{3}(0001)$ substrates in a high-vacuum chamber maintained at $3 \times 10^{-8}$ Torr by a turbo molecular pump. A diode-pumped solid-state laser (355 nm wavelength, $15 \mathrm{~ns}$ pulse width) running at $10 \mathrm{kHz}$ was used to ablate the $\mathrm{ZnO}$ target, which was maintained at a distance of $50 \mathrm{~mm}$ from the substrate. The energy per laser pulse was fixed at $200 \mu \mathrm{J}$, and the laser beam scanned over the target surface was tightly focused so that the fluence was maintained at approximately $2 \mathrm{~J} / \mathrm{cm}^{2}$. The films were grown at a rate of approximately $4 \mathrm{~nm} / \mathrm{min}$ over a wide range of

*E-mail address: mychern@ccms.ntu.edu.tw substrate temperatures from 600 to $900^{\circ} \mathrm{C}$. Atomic oxygen was introduced into the chamber during the growth using a $\mathrm{RF}$ atom source operated at $300 \mathrm{~W}$, while the oxygen pressure was maintained from $1 \times 10^{-5}$ to $3 \times 10^{-4}$ Torr. The film quality was observed to strongly depend on the substrate temperature as well as the oxygen pressure. The characterizations of the optimized films grown at the substrate temperature of $800^{\circ} \mathrm{C}$ and oxygen pressure of $1 \times 10^{-5}$ Torr are as follows.

The crystal structure was investigated by high-resolution X-ray diffraction (HRXRD) using a parabolic Göbel mirror and a pair of channel-cut monochromating crystals in the $(+,-)$ setting. The total divergence of the $\mathrm{Cu} \mathrm{K} \alpha_{1}$ line is less than 10 arcsec. The $\theta-2 \theta$ scan of the optimized sample yields $5.2109 \AA$ for the lattice constant $c$ of the $\mathrm{ZnO}$ film. Compared to the bulk lattice constant, 5.2049 $\AA$, the film was found to be relaxed. The in-plane orientation relationship, $\mathrm{ZnO}[10 \overline{10} 0] \|$ sapphire[1120], was identified using the $\omega$ scan, where no other domains with different orientations could be detected. Figure 1(a) shows the detailed $\theta-2 \theta$ scan around the $\mathrm{ZnO}(0002)$ reflection, where persistent pendellösung fringes are clearly observed, indicating a uniform film with a negligible variation in lattice constant $c$. The thickness of the film obtained by fitting the period of the fringes is $128 \mathrm{~nm}$. Figure 1(b) shows the $\omega$-scan (rocking curve) of the $\mathrm{ZnO}(0002)$ reflection, where the narrow FWHM, $\sim 10 \operatorname{arcsec}$, approaches the resolution of the Xray machine, implying a very small tilt in the $c$ planes and perfect ordering along the growth direction. A similar result has been achieved by MBE growth, however a thin buffer layer of $\mathrm{MgO}$ was employed. ${ }^{7}$ Here, despite the large lattice mismatch, high-quality $\mathrm{ZnO}$ films were grown by FPLD directly on sapphire substrates without a buffer layer. Presumably, the growth mode in FPLD is two-dimensional layer-by-layer for $\mathrm{ZnO}$ to align perfectly with $\mathrm{Al}_{2} \mathrm{O}_{3}(0001),{ }^{7,10)}$ but this awaits further investigation.

Atomic force microscopy (AFM) was used to study the surface morphology of the films grown by FPLD and conventional PLD. For conventional PLD, a KrF excimer laser $(248 \mathrm{~nm}$ wavelength, $23 \mathrm{~ns}$ pulse width and $1 \mathrm{~Hz}$ repetition rate) was used, and the fluence was also maintained at $2 \mathrm{~J} / \mathrm{cm}^{2}$ on the target for comparison. The flat surface of the film grown by FPLD was confirmed by AFM measurement. The AFM image [Fig. 2(a)] of the FPLD film shows that the roughness of the surface is about $0.9 \mathrm{~nm}$ 

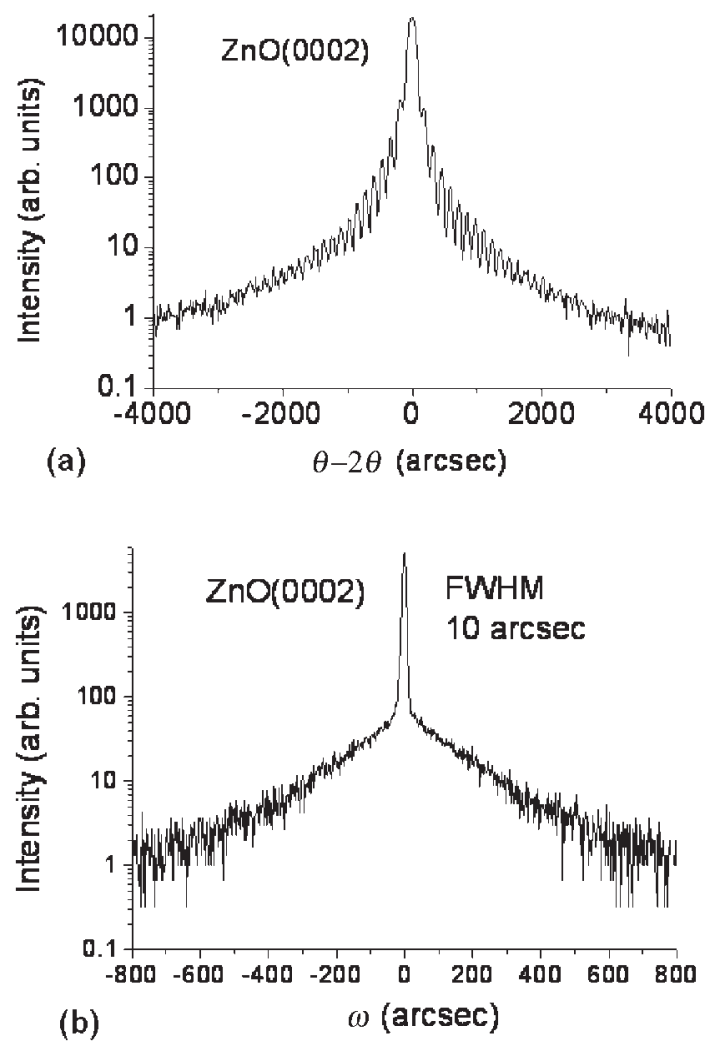

Fig. 1. (a) $\mathrm{X}$-ray $\theta-2 \theta$ scan of the $\mathrm{ZnO}(0002)$ reflection showing pronounced Pendellösung oscillations. (b) X-ray $\omega$-scan of the $\mathrm{ZnO}(0002)$ reflection showing a width of only 10 arcsec.

and no grain boundary can be clearly observed. Compared to the film grown by conventional PLD, with a (0002) reflection of more than 1000 arcsec typical FWHM, the roughness is about $5 \mathrm{~nm}$ and the grain size is about $200 \mathrm{~nm}$ for the boundary observed [Fig. 2(b)].

The optical emission properties were characterized by photoluminescence (PL) spectroscopy, excited by the $325 \mathrm{~nm}$ emission from a $20 \mathrm{~mW} \mathrm{He}-\mathrm{Cd}$ laser. The PL signal was collected using a PMT spectrometer with a resolution of $0.1 \mathrm{~nm}$. In general, the emission spectrum of $\mathrm{ZnO}$ can be divided into two broad categories: near band-edge (NBE) emissions $(\sim 3.36 \mathrm{eV})$ and deep-level (DL) emissions $(\sim 2.4 \mathrm{eV})$ observed in the films grown by other researchers. $^{7,12)}$ Notably, the DL emissions are almost not observed in the films grown by FPLD, indicating that the number of oxygen vacancies associated with the DL emissions can be sufficiently reduced by FPLD. Figure 3 shows the temperature dependence of the NBE emissions from 250 to $10 \mathrm{~K}$. At $10 \mathrm{~K}$, the dominant NBE peaks located at 3.365, 3.336, and $3.222 \mathrm{eV}$ were identified as the donor-bound exciton $\left(\mathrm{D}^{0}, \mathrm{X}\right){ }^{8,13)}$ two-electron satellite (TES), ${ }^{8,14)}$ and donoracceptor pair transition $\left(D^{0}, A^{0}\right),{ }^{8,13)}$ respectively. The most intense peak $\left(\mathrm{D}^{0}, \mathrm{X}\right)$ has an FWHM less than $5 \mathrm{meV}$. All these peaks have phonon replicas involving multiples of an LO phonon of $\sim 72 \mathrm{meV}^{15}$ ) located at 3.294, 3.193, and $3.150 \mathrm{eV}$, respectively. In addition, there are four shoulders on the right side of the $\left(\mathrm{D}^{0}, \mathrm{X}\right)$ peak, which were identified as the A-band free exciton (FXA), ${ }^{16)}$ B-band free exciton $(\mathrm{FXB}),{ }^{16)}$ high-order FXA $(n=2)$ and high-order FXB $(n=2)$. The binding energies of the A-band and B-band free excitons are approximately 36 and $62.7 \mathrm{meV}$, respec-
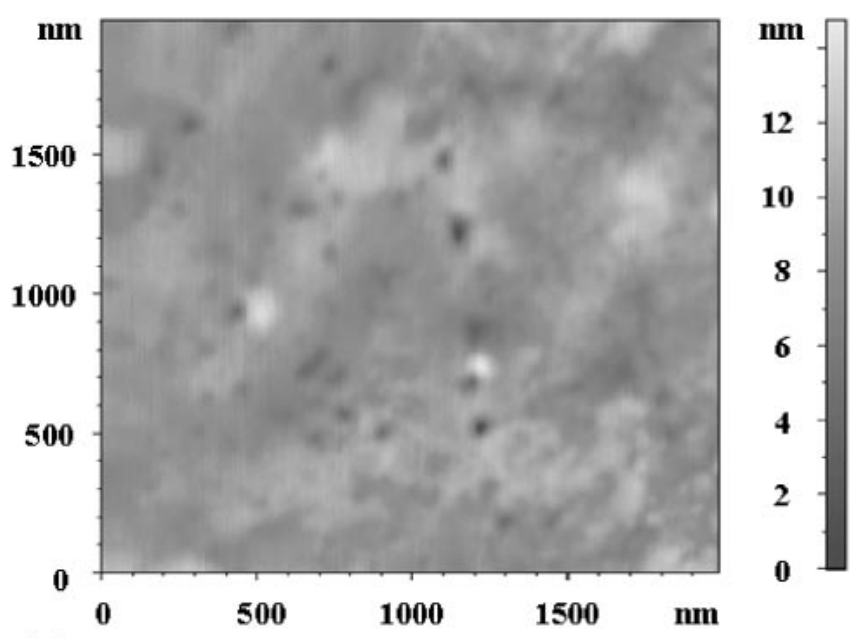

(a)
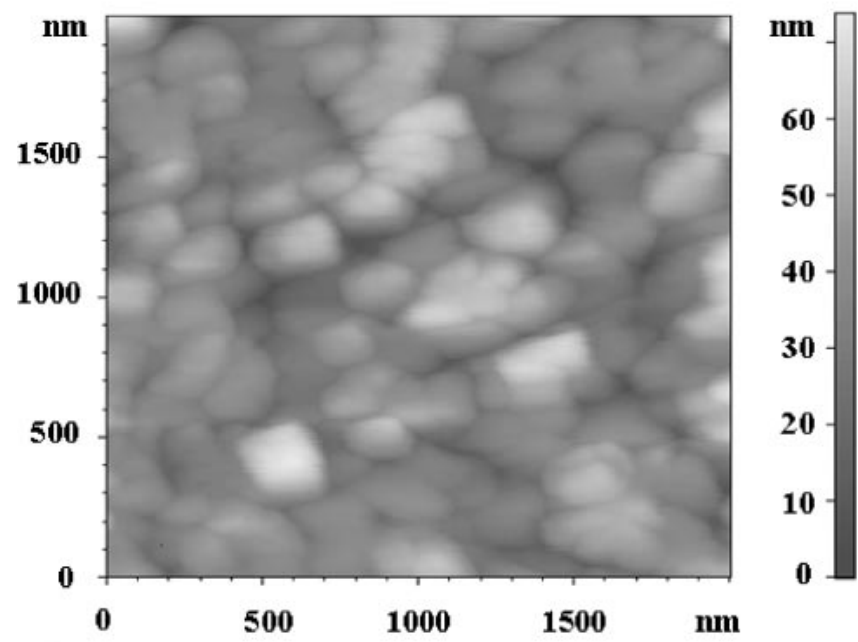

(b)

Fig. 2. AFM images for films grown by (a) FPLD and (b) conventional PLD.

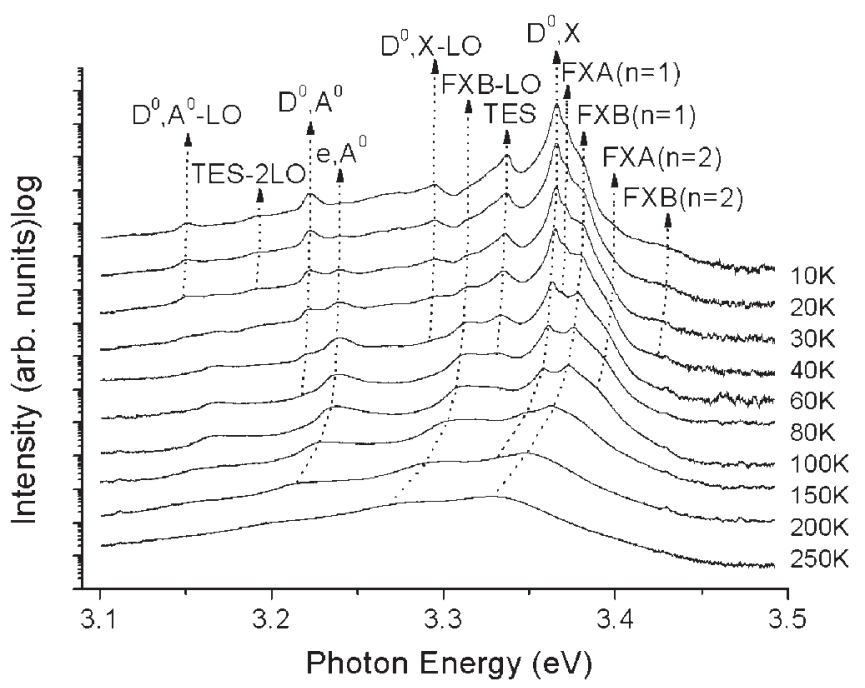

Fig. 3. PL NBE emissions of the $\mathrm{ZnO}$ film from 250 to $10 \mathrm{~K}$. Fine structures of exciton states with phonon replicas and high-order free exciton states are well resolved. 
tively, which were calculated using the formula: $E_{\mathrm{b}}=$ $4 / 3\left[E_{\mathrm{FX}}(n=2)-E_{\mathrm{FX}}(n=1)\right]$, and are in agreement with those previously reported. ${ }^{9,14)}$ The high-order free excitons are observed to last up to $40 \mathrm{~K}$. These PL properties demonstrate the high quality of the $\mathrm{ZnO}$ films grown by FPLD.

The Hall properties of $\mathrm{ZnO}$ films were studied by the standard four-point Van der Pauw technique, in which the Ohmic contact was achieved using annealed $\mathrm{Au}$ electrodes. ${ }^{16,17)}$ The typical intrinsic $\mathrm{ZnO}$ film prepared by PLD is of the n-type with a carrier concentration of $10^{18} \mathrm{~cm}^{-3}$, a resistivity on the order of $10^{-1} \Omega \mathrm{cm}$, and an electron mobility of $10 \mathrm{~cm}^{2} / \mathrm{V} \mathrm{s}$ due to various defects. ${ }^{4,6)}$ $\mathrm{ZnO}$ films grown by FPLD achieve a high mobility up to $100 \mathrm{~cm}^{2} / \mathrm{V} \mathrm{s}$ at $300 \mathrm{~K}$ with a carrier concentration in the range from 2 to $5 \times 10^{16} \mathrm{~cm}^{-3}$, indicating that the number of intrinsic defects of the films can be reduced by FPLD.

In conclusion, an FPLD method was developed for thin film synthesis, and $\mathrm{ZnO}$ films were grown on $\mathrm{Al}_{2} \mathrm{O}_{3}(0001)$ substrates by this new method without a buffer layer. The structural, optical, and electrical properties show the high quality of the films. We expect that the FPLD method will be successful not only for the growth of pure $\mathrm{ZnO}$ films, but also for the doping of $\mathrm{ZnO}$ films and the growth of many other crystals.

\section{Acknowledgements}

This work is supported by the National Science Council of Taiwan. In addition, the authors are grateful for the financial support from the Ministry of Economic Affair of Taiwan, under the grant number 93-EC-17A-08-S1-0006.
1) Y. R. Ryu, T. S. Lee, J. H. Leem and H. W. White: Appl. Phys. Lett. 83 (2003) 4032

2) X.-L. Guo, J.-H. Choi, H. Tabata and T. Kawai: Jpn. J. Appl. Phys. 40 (2001) L177.

3) Y. I. Alivov, E. V. Kalinina, A. E. Cherenkov, D. C. Look, B. M. Ataev, A. K. Omaev, M. V. Chukichev and D. M. Bagnall: Appl. Phys. Lett. 83 (2003) 4719.

4) S. Choopun, R. D. Vispute, W. Noch, A. Balsamo, R. P. Sharma, T. Venkatesan, A. Iliadis and D. C. Look: Appl. Phys. Lett. 75 (1999) 3947.

5) Y. R. Ryu, S. Zhu, J. D. Budai, H. R. Chandrasekhar, P. F. Miceli and H. W. White: J. Appl. Phys. 88 (2000) 201.

6) X.-L. Guo, H. Tabata and T. Kawai: J. Cryst. Growth 223 (2001) 135.

7) Y. Chen, H.-J. Ko, S.-K. Hong and T. Yao: Appl. Phys. Lett. 76 (2001) 559.

8) D. C. Look, D. C. Reynolds, C. W. Litton, R. L. Jones, D. B. Eason and G. Cantwell: Appl. Phys. Lett. 81 (2002) 1830.

9) A. Tsukazaki, A. Ohtomo, M. Kawasaki, T. Makino, C. H. Chia, Y. Segawa and H. Koinuma: Appl. Phys. Lett. 84 (2004) 3858.

10) J.-Y. Oh, J.-H. Lim, D.-K. Hwang, H.-S. Kim, R. Navamathavan, K.-K. Kim and S.-J. Park: J. Electrochem. Soc. 151 (2004) G623.

11) G. Xiong, J. Wilkinson, B. Mischuck, S. Tüzemen, K. B. Ucer and R. T. Williams: Appl. Phys. Lett. 80 (2002) 1195.

12) T. Matsumoto, H. Kato, K. Miyamoto, M. Sano, E. A. Zhukov and T. Yao: Appl. Phys. Lett. 81 (2002) 1231.

13) Y. R. Ryu, T. S. Lee and H. W. White: Appl. Phys. Lett. 83 (2003) 87.

14) K. Thonke, Th. Gruber, N. Teofilov, R. Schönfelder, A. Waag and R. Sauer: Physica B 308-310 (2001) 945.

15) D. C. Reynolds, D. C. Look, B. Jogai and H. Morkoç: Solid State Commun. 101 (1997) 643.

16) Y. R. Ryu, W. J. Kim and H. W. White: J. Cryst. Growth 219 (2000) 419.

17) M. Kurimoto, A. B. M. Almamun Ashrafi, M. Ebihara, K. Uesugi, H. Kumano and I. Suemune: Phys. Status Solidi B 241 (2004) 635. 